\title{
LITIASIS ÓSEA: PATOLOGÍA EXCEPCIONAL
}

\author{
J.G. PEREIRA ARIAS*, J.G. IBARLUZEA GONZÁLEZ**, J.A. ÁLVAREZ MARTÍNEZ*** \\ *Servicio de Urología del Hospital de San Eloy. ${ }^{* *}$ Servicio de Urología y ***Anatomía Patológica del Hospital de \\ Galdakao. Bizkaia.
}

Actas Urol Esp. 27 (6): 471-477, 2003

\section{RESUMEN}

\section{LITIASIS ÓSEA: PATOLOGÍA EXCEPCIONAL}

La Litiasis ósea constituye una entidad excepcional de escaso impacto en la literatura médica. Su origen asienta sobre la metaplasia ósea del urotelio y conjuntivo subyacente. Su diagnóstico requiere un alto índice se sospecha donde los signos radiológicos y la resistencia a la litotricia extracorpórea pueden orientar sobre ésta rara patología. No obstante, el diagnóstico de certeza lo establece el estudio histológico.

Presentamos un paciente de 49 años con uropatía obstructiva izquierda secundaria a litiasis ureteral lumbar alta que precisó ureterolitectomía y resección segmentaria del uréter tras fracaso de la litotricia extracorpórea. El diagnóstico derivó del estudio histológico confirmando la presencia de litiasis ósea y metaplasia escamosa. Revisamos aspectos epidemiológicos, diagnósticos y terapéuticos en la literatura. El tratamiento de la litiasis ósea requiere la resección del cálculo y del urotelio subyacente en aras a prevenir la recidiva de la misma. De igual modo, la posible asociación a degeneración tumoral nos sugiere un adecuado control evolutivo.

PALABRAS CLAVE: Litiasis ósea. Metaplasia ósea.

\section{ABSTRACT \\ BONE LITHIASIS: UNCOMMON PATHOLOGY"}

Bone lithiasis is an uncommon disease unfrequent reported in the literature. Its origin is supported on urothelium and conjuctive tissue bone metaplasia. High suspiccius index is needed to establish its diagnosis; where radilogical signs and extracorporeal lithotripsy resistence drive to these uncommon entity. Definitive diagnosis is based on hystological exam.

We report a 49-years-old patient with left obstructive uropathy due to a high ureteral lumbar lithiasis who precised ureterolithectomy and parcial ureterectomy after extracorporeal litotripsy failure. Hystologycal exam confirmed bone lithiasis and scamous metaplasia. Epidemyologic, diagnostic and therapeutical aspects are briefly reviewed in literature. Bone calculous treatment include lithectomy and urothelium resection to avoid posterior recurrence. Posible association to tumoral transformation suggest close evolutive follow-up.

KEY WORDS: Bone lithiasis. Bone metaplasia. 
$\mathrm{L}^{\mathrm{a}}$ a presencia de tejido óseo en el aparato urinario aunque infrecuente, ha sido referida en la literatura médica. La litiasis ósea viene definida por la agregación cristalina que ocurre sobre un foco de metaplasia ósea del urotelio o conjuntivo subyacente $^{1-4}$. Su incidencia oscila entre 0,28 y el $1,16 \%$ del conjunto de litiasis ${ }^{3-6}$. No obstante, su diagnóstico es subestimado por no estar los urólogos "familiarizados" con esta entidad. De manera adicional el advenimiento de la LEOC como modalidad terapéutica aplicable al 90\% de las litiasis, ha permitido la resolución de estas sin poder conocer la historia natural evolutiva del cálculo al no poder conocer la composición del núcleo sino de los fragmentos expulsados resultantes de la litotricia. Por ello, se requiere un alto índice de sospecha para orientar el diagnóstico de la litiasis ósea y arbitrar su adecuado tratamiento.

La capacidad osteogénica del urotelio es conocida desde hace mucho tiempo. Las osificaciones renales descritas en ausencia de litiasis, se originan en focos con la constante participación del urotelio de los cálices y pelvis renal ${ }^{7}$. La primera referencia de una litiasis ósea se atribuye, hasta fechas recientes a Phemister, quién describe 3 cálculos renales en dos pacientes en $1923^{8}$. No obstante, actuales investigaciones atribuyen a JB Trehet esta primicia por su descripción de un cálculo renal con puntos óseos en 1812, pasando a ser el primer autor en describir una litiasis ósea 9 . La primera referencia de cálculo óseo a nivel vesical fue descrita por Cifuentes en un estudio retrospectivo del análisis de 828 cálculos recogidos entre 1913 y $1973^{1,2}$. De igual modo, Sharp y Houstet describen piedras enquistadas $\mathrm{y}$ adherentes a la vejiga como un hueso "careado". De manera adicional, han sido descritas litiasis óseas a nivel ureteral como el caso que nos ocupa $^{3,10,11}$.

\section{CASO CLÍNICO}

Presentamos un paciente de 49 años de edad con antecedentes personales de salmonelosis y trombopenia familiar, remitido a nuestra Unidad de Litotricia por hallazgo casual en estudio radiológico de litiasis ureteral lumbar izquierda. El estudio ecográfico objetivó la presencia de ureteropielocaliectasia izquierda grado II-III y parénquima parcialmente conservado con un espesor variable de 1,6 a 2,2 cms. La urografía intravenosa constató la severa dilatación pielocalicial secundaria a uropatía obstructiva incompleta por litiasis de $2 \times 1,5 \mathrm{~cm}$ a nivel ureteral lumbar L3 con densidad inhomogénea y área hipodensa excéntrica periférica; así como pequeños cálculos en grupo caliciliar inferior (Figs. 1 y 2). El estudio analítico aportado reflejó una insuficiencia renal con creatinina plasmática de $2,4 \mathrm{mg} / \mathrm{dl}$ y trombopenia (97.000 plaquetas/ml).

Se realizó Litrotricia extracorpórea mediante Litotriptor Dornier Compact Delta previo posicionamiento de nefrostomía percutánea ecodirigida. En el control radiológico post-LEOC a la semana, se objetivó fragmentación y calle litiásica a nivel pelviano y lumbar alto (Fig. 3). Dada la eficacia fragmentadora se practicó una segunda sesión de LEOC permitiendo la reducción del volumen litiá-

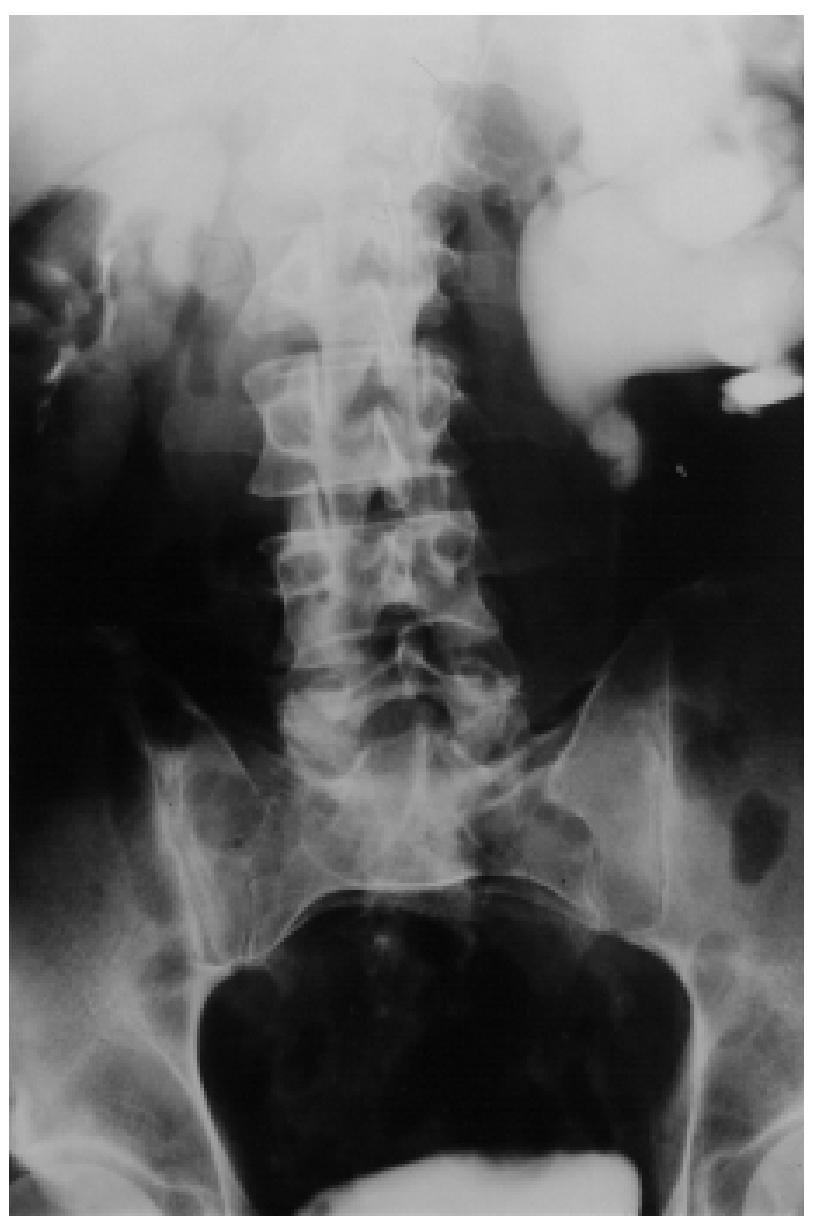

FIGURA 1. Urografia intravenosa: severa ureteropielocaliectasia izquierda secundaria a uropatia obstructiva incompleta por litiasis a nivel ureteral lumbar L3. 


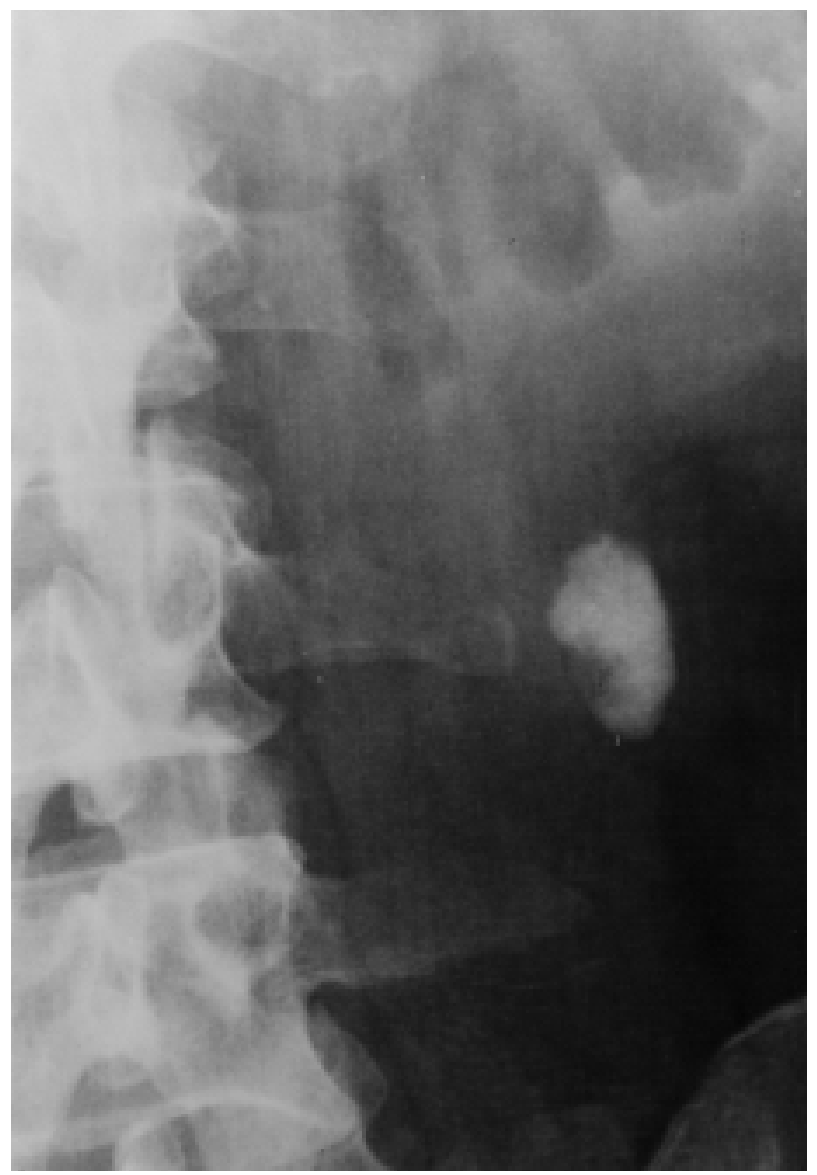

FIGURA 2. Rx simple aparato urinario: cálculo ureteral lumbar alto, de $2 \times 1,5 \mathrm{~cm}$, de densidad inhomogénea y área hipodensa excéntrica periférica medial.

sico y la posterior expulsión de los cálculos fragmentados tras oclusión de la nefrostomía. En pielografía anterógrada, se confirmó la permeabilidad de la vía urinaria y la extraordinaria firmeza del fragmento residual a la pared urotelial. La creatinina plasmática se normalizó en cifras de $1,13 \mathrm{mg} / \mathrm{dl}$ tras el episodio obstructivo agudo. Los fragmentos expulsados y analizados presentaron una composición mixta de oxalato cálcico monohidrato y fosfato cálcico. Animados por los resultados, realizamos una tercera sesión de litotricia extracorpórea que resultó completamente infructuosa.

Dada la ineficacia de la LEOC en resolver el fragmento residual, la extremada fijeza de la litiasis a la pared ureteral y bajo la sospecha de litiasis adherida al urotelio, se ofreció cirugía abierta o endoscópica (ureterolitectomía percutánea). Se realizó ureterolitectomía y resección segmentaria del uréter lumbar con ureteropieloplastia desmembrada sobre catéter ureteral doble $J$, así como extracción de los fragmentos residuales alojados en cáliz inferior. El postoperatorio transcurrió sin incidencias retirando el tutor ureteral a las 6 semanas. El resultado histológico reportó la presencia de una formación calculosa pardo-oscura de $1 \mathrm{~cm}$ de diámetro intimamente adherida a la mucosa sobre área excavada en la pared ureteral. La unión pieloureteral presentaba un foco de ulceración con acumulo de material cristaloide y reacción gigantocelular a cuerpo extraño, así como áreas de metaplasia escamosa. El calculo presentaba una zona central de tejido óseo con trabeculas gruesas y espacios medulares con abundantes vasos y fibrosis irregular compatible con litiasis ósea (Figs. 4 y 5).

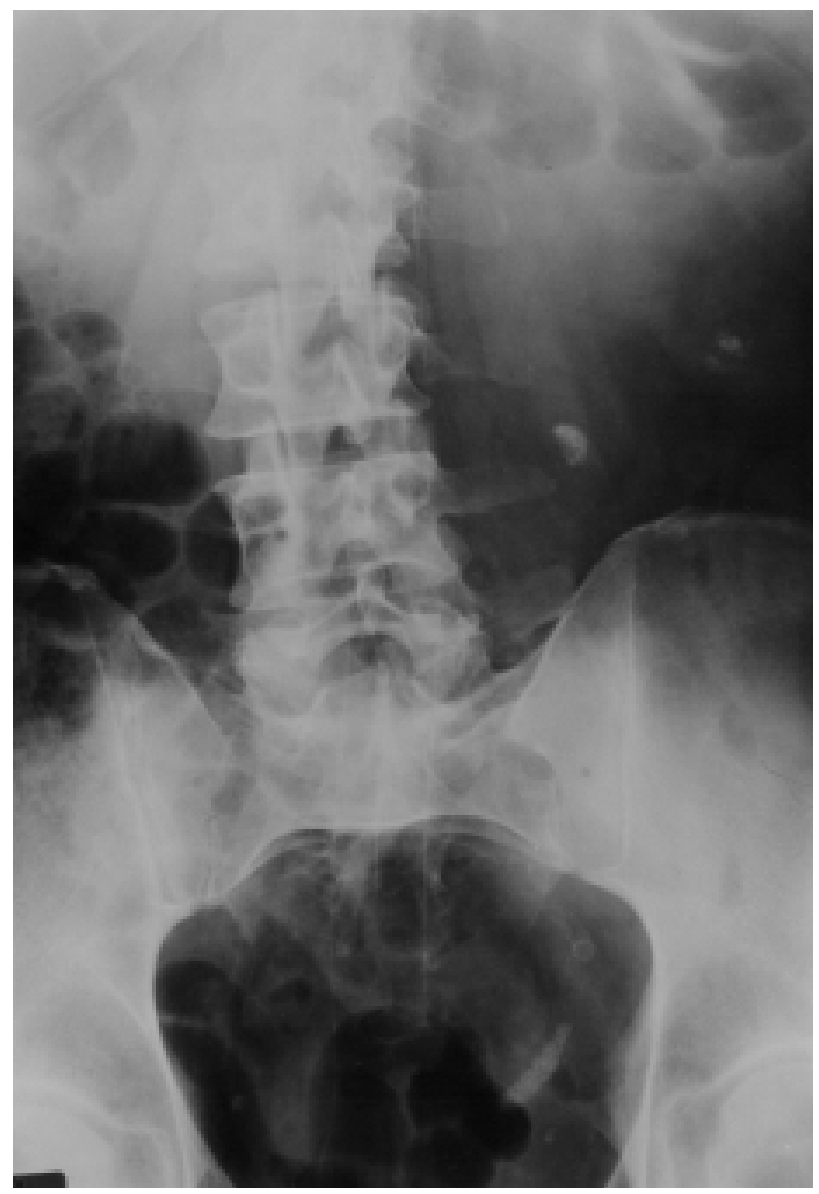

FIGURA 3. Fragmentación post-ESWL y calle litiásica ureteral pelviana de la porción cristalina del cálculo, con persistencia litiásica lumbar alta. 


\section{DISCUSIÓN}

La aparición de tejido óseo en órganos que en condiciones normales no lo presenta es frecuente; así se ha descrito hueso ectópico en aparato digestivo, traquea, sistema genitourinario, paredes arteriales, ojos, músculos y tendones. Este fenómeno de metaplasia ósea, definida como la sustitución adaptativa de una célula adulta diferenciada por otra célula adulta (osteocitos, osteoblastos y osteoclastos) suele acontecer en células epiteliales y del tejido conectivo. El proceso, similar a la osificación intramembranosa, requiere un estímulo regenerativo inductor similar al que acontece en un proceso de cicatrización tras una lesión en tejidos blandos, donde puede aparecer una zona ósea en la lesión, fruto de la metaplasia de fibroblastos a osteoblastos.

Han sido diversos autores los que han mostrado inquietud sobre la litogénesis ósea. Se esgrimen diversas teorías etiopatogénicas sin poder definir en la actualidad la causa o causas que expliquen su aparición. Así se citan diversos estudios experimentales que pretenden arrojar información sobre su origen. Al parecer diversos tipos de tejido conjuntivo tiene potencialidades osteogénicas latentes que se manifiestan raramente. Esta conclusión viene avalada por la producción experimental de metaplasia ósea tras la ligadura de las arterias y venas renales ${ }^{12,13}$ y después de una variedad de manipulaciones experimentales como: implante de cartílago xifoides asociado a la inyección subcutánea de sales cálcicas $^{14}$, injertos subcutáneos de mucosa vesical y de pelvis renal ${ }^{15}$, transplante de mucosa vesical en la vaina de los músculos rectos abdominales ${ }^{16}$ y tras implante de aponeurosis de los rectos del abdomen en la mucosa vesical ${ }^{5}$. De igual modo, la inyección de extractos alcohólicos en músculo puede inducir la osteogénesis, y compartir esta capacidad con otros irritantes químicos ${ }^{17}$. Así, la inyección del carcinógeno 20-metilcolantreno en la pared ureteral induce la metaplasia a tejido óseo esponjoso ${ }^{18}$.

La osificación ectópica precisa colágeno, mucoproteínas que proporcionen la matriz orgánica, fosfato cálcico del tipo de la hidroxiapatita y tejido conjuntivo y vasos. Se sugiere la posibilidad de que las placas de Randall o concreciones de Carr de las papilas generen una zona de lesión-necrosis, que induce una transformación metaplásica del tejido lesionado y permite la posterior fijación de cristales de oxalato cálcico. La fase de mineralización previa a la osificación viene determinada por el depósito de fosfato cálcico apatítico, sustancia presente en todas las calcificaciones patológicas del organismo. Este proceso es consecuencia del estímulo producido por la actividad enzimática de las fosfatasas alcalinas liberadas en el epitelio urinario y por los factores locales producidos en los fibroblastos del tejido conjuntivo adyacente (IGF, Beta-TGF) en íntima conexión con el colágeno local y los capilares ricos en inductores de osificación (osteotaxina) que surgen de la mucosa de los túbulos excretores. Estos factores contribuyen a la diferenciación celular y a la aparición de células de la línea osteoblástica. Se cree que los osteoblastos derivarian de las células endoteliales de los capilares preformados o bien de las células del mesénquima perivascular. Estas células sintetizan colágeno tipo I, osteocalcina, glicosaminglicanos y proteogliconos que constituyen la matriz osteoide, que será mineralizada por la acción de las fosfatasas alcalinas del urotelio generando las trabéculas óseas. A continuación sobre el núcleo óseo se depositarian sales de oxalato cálcico monohidrato, por ser la sustancia con mayor facilidad precipitan sobre un núcleo patológico, permitiendo la posterior agregación y crecimiento del cálculo. Si el cálculo se desprende y pierde el contacto con el urotelio se origina una pérdida de nutrición permitiendo la necrosis de los osteocitos conservándose las trabéculas y lagunas óseas. Por ello, en las litiasis óseas ureterales es infrecuente identificar osteocitos en las trabéculas óseas ${ }^{1,3,4,19-22}$.

Desde el punto de vista epidemiológico, se han descrito alrededor de 60 casos de litiasis ósea en la literatura (54 casos hasta 1995). Su incidencia se cifra entre $0,28-1,16 \%$, aunque probablemente sea más cercana al $2 \%$ del total de las litiasis por hallarse esta infravalorada ${ }^{1,3,5}$. Las series más amplias son de procedencia nacional y destacan los 19 cálculos de Cifuentes ${ }^{3}$, 15 cálculos en 14 pacientes de Lovaco $^{5}$ y los 7 casos de García-Cuerpo ${ }^{20}$. De la revisión de estas series se desprende que no existe predominio de sexo ni de 
lateralidad, afectando casi por igual a ambas unidades renoureterales. Su incidencia se distribuye entre los 23 y 66 años, con mayor presencia entre los 40-50 años ${ }^{3,5,20}$.

Desde el punto de vista clínico, la litiasis ósea no difiere en su forma de presentación del resto de la patología litiásica. Así se han descrito casos de anulación renal e hidronefrosis, sepsis urinaria, crisis renoureteral y hallazgos incidentales. No obstante, algunos autores refieren que es más frecuente la presencia de algias lumbares que el típico cólico nefrítico ${ }^{5}$. El escenario analítico bioquímico y hematológico no arroja datos significativos. La citología urinaria suele ser inespecífica; no obstante la posible asociación de la metaplasia ósea con tumores del urotelio o su posterior degeneración sarcomatosa realzan la importancia de su utilidad en el seguimiento evolutivo de estos pacientes ${ }^{5,23,24}$. En nuestro paciente la presentación fue silente y resultó hallazgo incidental en estudio ultrasónico abdominal. La presencia de metaplasia escamosa, hallada en nuestro paciente, nos alerta sobre la posible degeneración a carcinoma epidermoide, como ocurre ante procesos irritativos crónicos sobre el urotelio.

El diagnóstico de sospecha debe sustentarse en los hallazgos radiológicos característicos ${ }^{20}$. Así, en la radiografía simple abdominal podemos encontrar cálculos de densidad inhomogénea, de morfología reniforme irregular, con una zona excéntrica de menor densidad radiológica rodeada, aunque no en su totalidad, por una imagen litiásica de alta densidad. La zona de menor densidad corresponde al pedículo metaplásico que sirve de anclaje al urotelio. A tenor de los resultados de la revisión bibliográfica, podemos afirmar que las localizaciones más frecuentes de la litiasis ósea en orden decreciente son las siguientes: unión pieloureteral, pelvis renal, cáliz inferior, cáliz superior, cáliz medio, vejiga y uréter ${ }^{3-}$ $5,19,20$. Otro rasgo distintivo de este grupo litiásico viene definido por la desproporción existente entre las características del cálculo y la alteración morfofuncional que origina en el estudio mediante urografia intravenosa. De igual modo, es llamativa la extraordinaria fijeza del cálculo al urotelio, tal y como se pone de manifiesto en la pielografía anterógrada o retrógrada. En la litiasis ósea es casi siempre la zona adherida y de menor densidad radiológica la que "mira" hacia el urotelio. Por el contrario los cálculos radiotransparentes de ácido úrico no suelen mostrar esta característica adherencia al urotelio, a excepción de precipitar sobre una lesión tumoral subyacen$\mathrm{te}^{4,20}$.

$\mathrm{Si}$ las características radiológicas anteriormente descritas, no alertan nuestro instinto diagnóstico escasamente familiarizado con esta patología, debemos sospechar la presencia de cálculo óseo ante la resistencia manifiesta a la fragmentación durante la LEOC sobre el área excéntrica de inferior densidad radiológica. Ante su sospecha debemos rechazar la LEOC y optar por un tratamiento quirúrgico (endourológico o abierto) que actúe sobre el cálculo y su base de implantación. La mayoría de autores destaca la necesidad de actuar sobre el foco de metaplasia ósea de la base de implantación del cálculo, en aras a confirmar su diagnóstico y prevenir futuras recidivas y la posible degeneración sarcomatosa o asociación a carcinoma urotelial ${ }^{3,5,19,20,23,24}$. En nuestro paciente, el diagnóstico de presunción aconteció tras el fracaso de la LEOC en resolver el fragmento residual adherido al urotelio, fruto de nuestra escasa familiaridad con esta entidad. Al paciente se le ofreció ureterolitectomía percutánea con objeto de resolver por vía endoscópica la litiasis caliciliar inferior y el cálculo ureteral. No obstante, la sospecha de litiasis ósea y la posibilidad de no poder resolver con éxito la base de implantación nos orientó hacia la cirugía abierta.

El diagnóstico definitivo lo determina el estudio histológico, que ratifica la presencia de tejido óseo en el cálculo o en su base de implantación. El cálculo se halla fijo al urotelio por el foco de metaplasia ósea, que suele presentar una característica "oquedad" en su interior ${ }^{1,3-5,20}$. Las técnicas de análisis del cálculo recomendadas son el procesado del cálculo por el método petrográfico de lámina delgada tras su decalcificación y posterior tinción, observación del mismo bajo microscopio de luz polarizada y el estudio histopatológico con tinción de hematoxilina-eosina y tricrómico de Masson 1,5,19,25. Al microscopio de luz polarizada a 100 aumentos, el tejido óseo presenta un color azul pálido. El espectrofotograma de energía dispersa por RX presenta un pico característico de hueso ${ }^{5}$. En microscopía electró- 


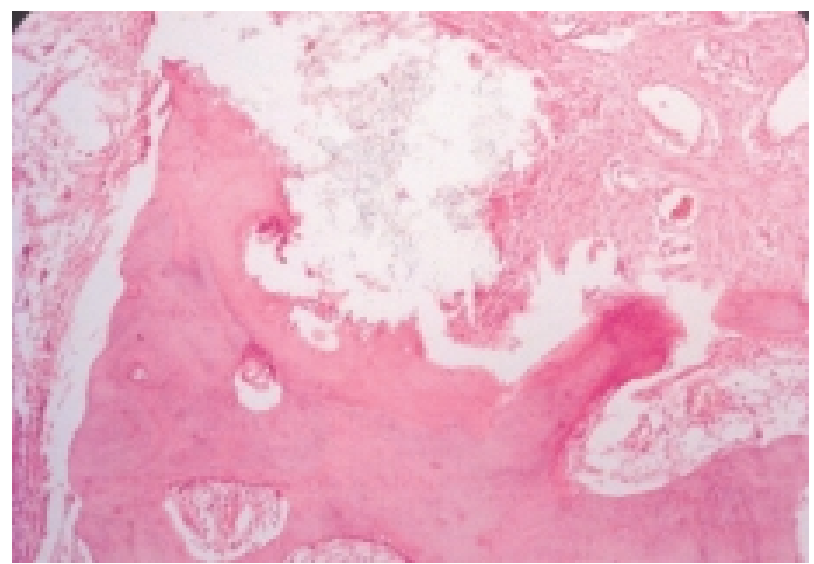

FIGURA 4. Zona central de tejido óseo con trabéculas gruesas y espacios medulares con abundantes vasos y fibrosis irregular compatible con litiasis ósea.

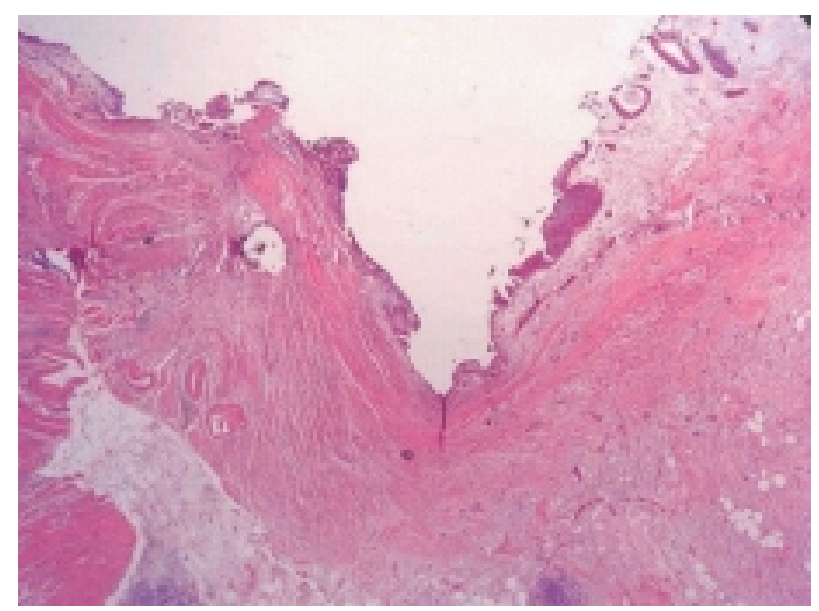

FIGURA 5. Mucosa transicional de unión ureteropiélica con área de ulceración y puntos de metaplasia escamosa. Prominente edema, fibrosis e inflamación en el corion subyacente.

nica se evidencian las trabéculas óseas y lagunas donde se alojan los osteoblastos. La porción cristalina inorgánica se presenta, en orden decreciente de frecuencia como: oxalato cálcico monohidrato, oxalato cálcico monohidrato asociado a fosfato cálcico y oxalato cálcico dihidrato ${ }^{3,5}$.

A modo de conclusión, la presencia de litiasis de densidad inhomogénea, con una zona excéntrica de menor densidad radiológica firmemente adherida al urotelio, con llamativa desproporción entre las características del cálculo y la alteración morfofuncional originada y resistente a la litotricia extracorpórea; nos debe alertar sobre la posibilidad de un cálculo óseo y arbitrar las medidas oportunas para su exéresis y la de su base de implantación. De igual modo, se aconseja un seguimiento citológico posterior ante la eventual posibilidad de degeneración y/o asociación a procesos neoplásicos.

\section{REFERENCIAS}

1. CIFUENTES L, GARCÍA E, VELA R, MIÑÓN JR.: Tejido óseo en cálculos del aparato urinario (cinco observaciones). Arch Esp de Urol 1973; 26 (5): 457480.

2. CIFUENTES L, RAPADO A, SANTOS M, TRABA ML.: Osseous and proeosseous structures in calculi of the urinary tract. Rev Clínica Esp 1973; 131 (3): 181-186.

3. CIFUENTES L, MINOR JL, SANTOS M, TRABA ML.: Ectopic renal ossification as nucleous of urinary stones. J Urol 1976; 116 (4): 398-401.

4. GARCÍA E, ROMERO C, REY A, GARCÍA R, LLORENTE C et al.: Bone lithiasis or bone metaplasia? Eur Urol 1984; 10 (4): 286-288.

5. LOVACO F, GARCÍA E, FERNÁNDEZ I, SERRANO A, GARCÍA R.: Litiasis ósea: histopatología experimental y clínica. Arch Esp de Urol 1994; 47 (2): 111-119.

6. FERNÁNDEZ M, CARALPS A, GIL-VERNET JM.: Tejido óseo en cálculos renales. Rev de la Real Academia de Barcelona 1986; 2: 43.

7. SALKI JO, ABESHOUSE BS.: Calcification, ossification and cartilage formation in the kidney. AJR Rad Therapy \& Nuclear Med 1962; 88: 125.

8. PHEMISTER DB.: Ossification in kidney stones attached to the renal pelvis. Ann Surg 1923; 78: 239.

9. LOVACO F, GARCÍA E, FERNÁNDEZ I, SERRANO A, NORÓN MV.: La litiasis ósea en el periodo de la Ilustración: D.F.J.G. Trehet. Arch Esp de Urol 2001; 54 (4): 297-310.

10. KLINGER ME.: Bone formation in the ureter. A case report. J Urol 1956; 77: 793.

11. SCHULMAN CC, WEISER M.: Formation osseuse pyélique. Acta Urol Belg 1971; 39: 322.

12. BLESSIG C.: Über die Veränderugen der Niere nach Unterbindung der Nierenarterie. Virchows Arch 1859; 16: 120 .

13. SACERDOTI C, FRATTIN G.: Ueber die heteroplastiche Knochenbildung. Virch Arch Path Anat Physiol 1902; 168: 431.

14. BOICHI A, DOCK W.: Experimental studies on heteroplastic bone formation. J Exp Med 1920; 32: 745.

15. HUGGINS CB.: The formation of bne under the influence of epiyhelium of the urinary tract. Arch Surg 1933; 27: 203.

16. ABBOTT AC, GOODWIN AM, STEPHENSON E. Heterotopic bone formation produced by epithelial transplants from urogenital tract of dogs, rabbits, guinea pigs and cats. $J$ Urol 1938; 40: 294.

17. HEINEN JH, DABBS GH ; MASON HA: The experimental production of ectopic cartilage and bone in the muscles of rabbitts. J Bone \& Jount Surg 1949; 31: 765-772. 
18. REY A: Osificación heterotópica del aparato urinario; tesis doctoral. Madrid, 1959.

19. ALCOVER J, FERNÁNDEZ M, CARRETERO P. Noyaux d'ossification dans les calculus du rein. Ann d'Urologie 1995; 29: 333-336.

20. GARCÍA E, LOVACO F, BERENGUER A, GARCÍA R.: Bone metaplasia in the urinary tract: a new radiological sign. J Urol 1988; 139 (1): 104-108.

21. KAWAGA S.: Enzyme histochemistry of bone induction by urinary bladder epiyhelium. J Histochen Cytochen 1964; 13: 255.

22. CANALIS E, McCARTHY T, CENTRELLA M. Growth factors and the regulation of bone remodeling. J Clin Invest 1988; 81: 277.

23. POBIL M, GONZÁLEZ J.: Metaplasia ósea en tumor de urotelio superior. Un caso. Actas Urol Esp1978; 2: 305 .
24. MIRACCO C, SALVESTRINI F, CETTA F et al.: Bone mataplasia (bone lithiasis) in a papillary transitional carcinoma of the renal pelvis. Eur Urol 1988; 15: 156-158.

25. DÍAZ R, LEIVA O, CALVO M, CARVAJAL H, BOROBIA V.: Bone lithiasis. Actas Urol Esp 1977; 1: 115118.

Dr. J.G. Pereira Arias

C/ Francisco Macía, 3 - 3o B

48014 Bilbao (Vizcaya)

(Trabajo recibido el 26 de septiembre de 2002) 